\title{
La diversificación educativa y la \\ segmentación social. Signos de una \\ época de nuevas desigualdades
}

\section{Revista Colombiana \\ Artículo de reflexión $\quad \begin{aligned} & \text { de Educación, } \mathrm{N}^{\circ} 70 . \\ & \text { Primer semestre de } 2016\end{aligned}$ \\ Bogotá, Colombia.}

\author{
//Educational Diversification and \\ Social Fragmentation. Signs of a \\ Time of New Inequalities
}

\section{//A diversificação educativa e a segmentação social. Sinais de uma época de novas desigualdades}

Recibido: 13/04/2015 Evaluado: 03/08/2015

\section{Resumen}

Licenciada en Ciencias de la Educación. Máster en Ciencias Sociales con especialidad en Educación, CONICET/OEA. Profesora titular de la Universidad Nacional de San Luis. Integrante del Banco de Evaluadores de SECYT y ConEAu. Directora del Proyecto Reforma Educativa y Cambio Institucional: la MErcantilización Universitaria (UnsL). Directora del proyecto Apoyo al Sector Educativo del MERCOSUR (PASEM-SAN LUIS). Argentina. Correo electrónico: anamariacorti@gmail.com Profesora y licenciada en Ciencias de la Educación, Diploma Superior en Ciancias Sociales con mención en Gedtión de las Instituciones Educativas FLACSO. Magíster en Sociedad e Institucuines, doctoranda en Folosofía y Letras de la Universidad Nacional de Buenos Aires. Docente e investigadora de la Universidad Nacional de San Luis. Argentina. Correo electrónico: belengodino@gmail.com

Licenciada y profesora en enseñanza media y superior en Ciencias de la Educación (unsL). Diplomada Superior en Ciancias Sociales con mención en Gestión de las Instituciones Educativas (FlACSO). Maestranda de la carrera de Maestría y Espcialización en Educación Superior (unsL). Docente investigadora de la Universidad Nacional de San Luis. Argentina. Correo electrónico: marialujanmontiverosgarro@gmail.com

Transitamos tiempos en los que las instituciones educativas no solo se ven interpeladas en su mandato fundacional, vinculado a la formación de la subjetividad ciudadana, sino que atraviesan serias dificultades para cumplir con sus propósitos educativos.

La desigualdad en los resultados entre los sectores y grupos sociales más y menos favorecidos deja al descubierto que esta situación afecta especialmente a estudiantes en situación de mayor desventaja o vulnerabilidad (Echeita Sarrionandia, G. \& Duk Homad, C., 2008).

En este trabajo analizamos el surgimiento de nuevos formatos escolares en la provincia de San Luis. Damos cuenta de cómo se plasma en una política pública el énfasis puesto en la diversidad. Interpretamos que la diferenciación de dispositivos escolares permitiría la capacidad inclusiva de las poblaciones excluidas del sistema escolar, segmentando para ello la oferta educativa.

Los nuevos formatos escolares y los planes de políticas focalizadas se inscriben dentro de una política neoliberal que se apega a la diversidad como recurso que tiende a enriquecer la formación de la población que asiste a dichas instituciones. A nuestro entender estas creaciones escolares basadas en la diferenciación de las ofertas del sistema educativo público de San Luis (Argentina) provocan mayor segmentación, mayor diferenciación educativa, y aumentan aún más las desigualdades sociales de los usuarios, toda vez que garantizan espacios de ingreso pero con ofertas académicas no equivalentes.

\section{Abstract}

In the time we are living in, educational institutions are not only questioned about their foundational aim, linked to the education on citizen subjectivity, but have serious difficulties to achieve their educational purposes.

The inequalities in the results between wealthier and poorer social groups reveal that this situation affects specially students in greater disadvantage or vulnerabi-lity (Echeita Sarrionandia, G. \& Duk Homad, C., 2008).

\section{Palabras clave}

Diversificación educativa, desigualdades sociales, escuela autogestionada, escuela digital, segmentación social

\section{Keywords}

Educational diversification, social inequalities, self-managed schools, digital schools, social fragmentation

\section{Palavras chave}

Diversificação educativa, desigualdades sociais, escola autogestionada, escola digital, segmentação social 
In this paper, we analyze the rise of new kinds of school in the province of San Luis. We account for the way in which the emphasis on diversity is reflected in public policies, interpreting that the differentiation of school devices should allow for the inclusion of the excluded population from the school system, fragmenting for this aim the educational offer.

The new kinds of school and the focalized public policies are developed within a neoliberal policy that dictates that diversity is a resource that tends to enrich the education of people who attend these schools. It is our opinion that these schools based on the differentiation of the offer of the educational system of the province lead to more fragmentation, a greater educational differentiation, increasing even more the social inequalities among users, as they guarantee access, but with unequal academic offers.

\section{Resumo}

Percorremos tempos nos que as instituições educativas não só se veem interpeladas em seu mandato fundacional, vinculado à formação da subjetividade cidadão, mas que atravessam profundas dificuldades para cumprir com seus propósitos educativos.

A desigualdade nos resultados entre os setores e grupos sociais mais e menos favorecidos, deixa ao descoberto que essa situação afeta especialmente a estudantes em situação de maior desvantagem ou vulnerabilidade (Echeita Sarrionandia, G. \& Duk Homad, C., 2008).

Neste trabalho analisamos o surgimento de novos formatos escolares na província de San Luis. Damos conta de como se põe numa política pública a ênfase desde a diversidade. Interpretamos que a diferenciação de dispositivos escolares permitiria a capacidade inclusiva das povoações excluídas do sistema escolar, segmentando para isso a oferta educativa.

Os novos formatos escolares e os projetos de políticas focalizadas, se inscrevem dentro duma política neoliberal que se relaciona à diversidade, como recurso que tende a enriquecer a formação da povoação que assiste a essas instituições. A nosso entender as criações escolares baseadas na diferenciação das ofertas do sistema educativo público de San Luis (Argentina) provocam maior segmentação, maior diferenciação educativa, e aumentam mais as desigualdades sociais dos usuários, a sua vez que garantiram espaços de ingresso, mas com ofertas acadêmicas não equivalentes.

El artículo presenta algunas reflexiones que se desprenden del trabajo de indagación que venimos realizando en el marco del proyecto de investigación "Inclusión educativa en la formación docente inicial, continua y en servicio en el Mercosur ${ }^{11 "}$. El mismo tiene entre sus objetivos generales favorecer acciones en red de instituciones de educación superior de Paraguay, Argentina y Brasil para el estudio de la inclusión educativa en la formación docente inicial, continua y en servicio; propender a formar docentes que favorezcan la educabilidad en contextos diversos. Y como objetivos específicos: diagnosticar el estado de situación de la

1 Proyecto de investigación en el marco del Programa de Apoyo al Sector Educativo del Mercosur (Pasem). Países intervinientes: Argentina, Brasil y Uruguay. Institución co-solicitante en Argentina: Universidad Nacional de San Luis. 
formación docente inicial, continua y en servicio en relación con la inclusión educativa y las características que adopta el sistema de educación en cada territorio; planificar y ejecutar en red experiencias de inclusión educativa en la formación docente inicial, continua y en servicio de los países implicados.

Uno de los caminos que estimamos relevante con el fin de plantearnos la formación docente en relación con los procesos de inclusión educativa fue desplegar una línea de análisis acerca de las políticas educativas que en los últimos años han estado vinculadas desde el discurso de la inclusión. Nuestro interés se depositó en analizar las características que fue adoptando el sistema educativo del territorio provincial (San Luis) y la formación docente, es decir, comenzar a pensar qué sistema se está organizando desde las políticas públicas y qué lineamientos orientan la formación docente actual.

En este sentido, intentaremos describir y reflexionar sobre algunas de las políticas educativas que en la provincia de San Luis se enmarcan o vinculan desde el discurso oficial a procesos de inclusión educativa, poniéndolas en tensión con otras miradas sobre la inclusión social y educativa y también con elementos que describen el escenario educativo mismo de la provincia.

\section{Tensiones entre los procesos de inclusión y exclusión social en sociedades segmentadas}

Resulta importante explicitar algunos aspectos de la mirada que asumimos sobre los procesos de inclusión y exclusión social que iluminan la reflexión sobre las políticas educativas de la provincia de San Luis.

Las sociedades occidentales industrializadas durante una parte del siglo xx alcanzaron unos niveles de inclusión e integración social relativamente importantes. Esta situación hacía aparecer como viable y deseable un modelo de capitalismo social o capitalismo con Estado de bienestar. Pero una vez que entraron en crisis las condiciones materiales que hacían posible y sustentaban este modo de funcionamiento de la sociedad, fue notorio el efecto de la "vulnerabilidad de masas" (Castel, 1997).

A partir de la década de los setenta el rasgo excluyente pasó a ser la nota principal en la dinámica socioeconómica. Desempleo, precariedad laboral y pobreza se convirtieron en los elementos distintivos de la cuestión social. Mientras en el terreno europeo se expandía al máximo el denominado Estado de bienestar durante las tres décadas posteriores a la etapa de posguerra, en América Latina se hicieron algunos intentos de construcción de un Estado de bienestar, con mayores o menores grados de "éxito" dependiendo de cada país 
y sus particulares estructuras socioeconómicas. Este proceso se llevó a cabo con la ambigüedad y las marchas y contramarchas impuestas por las limitaciones y dificultades propias de países pobres y dependientes. Aun así, lo más grave fue que este estado social mínimo se desmanteló de forma rápida y radical a partir de la década de los setenta y más acentuadamente a partir de la década de los ochenta, en el auge del modelo neoliberalconservador de Reagan-Thatcher y la crítica situación de las economías de la región (Piola, 2000).

Esto quiere decir, entre otras cosas, que si, en América Latina durante la época de expansión del Ilamado Estado de bienestar había una intencionalidad política de incluir a los sectores sociales tradicionalmente excluidos, como parte de proyectos políticos que genéricamente recibieron el nombre de nacional-populares, hoy esta intención y esta promesa se han dificultado (Piola, 2000).

Sin duda, uno de las tensiones fundamentales en los crecientes procesos de exclusión es y ha sido la relación entre la educación y el trabajo. La necesidad de articular los sistemas educativos y de formación profesional por la urgencia que demandan las actuales condiciones del contexto de globalización y competitividad es un tema que debería ocupar los primeros lugares de la agenda política de los estados nacionales. Citando a Corti (2000) es relevante tener en cuenta los siguientes aspectos:

La importancia cada vez mayor de la educación basada en entrenamientos y desarrollo de habilidades para el mundo del trabajo; la dificultad de separar los temas de "educación general" de los de 2educación para el trabajo" [...]; la necesidad de lograr un sistema de formación profesional o de educación para el trabajo en general que posibilite reforzar "capacidades básicas" (lectura y comprensión de textos y resolución de problemas matemáticos); brindar conocimientos técnicos específicos y generales; trabajar en el mejoramiento de las competencias de carácter comportamental (tales como capacidad de trabajo en equipo, de comunicación, de adaptación a condiciones cambiantes). (p.133)

Encontramos, por supuesto, una estrecha relación entre la formación educativa brindada por las diversas instituciones escolares y los circuitos laborales por los que circulan aquellas personas alfabetizadas. El tema se agudiza cuando los individuos no cuentan con las posibilidades de acceder a ambos sistemas (formación y 
profesional) ya que a partir de las limitaciones que se les presenten se fisuran aún más las líneas de inclusión y de participación social. Frente a ese escenario es pertinente continuar hablando de exclusión social. Como bien afirma Corti "la pobreza y el desempleo son los síntomas más evidentes de la dinámica excluyente" (2000, p. 134).

Como ha señalado Castel (1997), en el mismo siglo en que se construye la condición salarial del trabajador, entendida como la estabilidad que brinda la protección social de los derechos de los trabajadores, se destruye dicha condición en la medida en que se precariza el empleo, se modifica el significado mismo del concepto de trabajo como consecuencia del proceso de flexibilización laboral, y se vulneran las condiciones de inclusión al mundo laboral. Las consecuencias son devastadoras dada la importancia de este espacio en la construcción de la subjetividad (Birgin, 2004).

En el caso argentino, el problema adquiere sus propias características de acuerdo con las particularidades del mercado local de trabajo, donde lo más habitual no es el desempleo de larga duración sino la inestabilidad laboral, a lo cual se suma la precariedad de los puestos de trabajo. Habitualmente a estos puestos acceden aquellas personas con menor nivel educativo y calificación. De este modo, se van configurando trayectorias laborales caracterizadas por una alta rotación entre puestos precarios, de bajos ingresos, poco calificados, de corta duración, intercalados con periodos de desempleo, subempleo e incluso de salida del mundo laboral como producto del desempleo (Kessler, 2004).

La persistencia de este fenómeno atraviesa ya a varias generaciones de personas que han crecido en familias cuya condición de exclusión les ha impedido completar su educación, acceder a un trabajo digno y a la seguridad social. Esta vivencia signada por el desmantelamiento social configura formas de subjetividad particular que deben ser consideradas por la magnitud de los sectores sociales a los cuales afecta, y el efecto desintegrador que producen, contrario a dinámicas sociales que propicien una ciudadanía amplia y participativa (Corti, 2008).

Es importante en este punto detenernos a revisar qué entendemos por procesos de exclusión social, para lo cual parecen muy potentes las categorías de Roberto Castel. El autor afirma que "la exclusión es inmóvil. Designa un estado o, más bien, estados de privación" (1997, p. 16). La categoría de desafiliación que presenta nos permite analizar en mayor profundidad las situaciones sociales actuales, ya que "no necesariamente equivale a una ausencia de vínculos, sino también a la ausencia de inscripción del sujeto en estructuras dadoras de sentido" (p. 421).

Es interesante, además, recuperar su planteo con relación a lo que él denomina "zonas" de cohesión social. Plantea zonas de integración social al establecerse una asociación 
entre trabajo estable/inserción sólida, es decir, cuando el individuo participa en las redes de sociabilidad. A la inversa, dice el autor, la ausencia de participación en alguna actividad productiva y el aislamiento relacional conjugan sus efectos negativos para producir la exclusión, o más bien, la desafiliación.

El modelo que propone Castel es interesante por cuanto no intenta ubicar a los individuos en esas "zonas" que se mencionaron, sino que intenta aclarar los procesos que llevan a los individuos de una zona a otra: por ejemplo pasar de la integración a la vulnerabilidad, o caer de la vulnerabilidad a la inexistencia social.

Por consiguiente, volver a poner en el centro del debate a la educación conlleva la responsabilidad de analizar las relaciones de un modelo de Estado que ha aumentado su función de control pero que muestra una débil capacidad de dinamizar las condiciones requeridas para asegurar prestaciones de calidad, al mismo tiempo en que fue desligándose de las responsabilidades de garantizarlas mediante vínculos con el sistema productivo, con el fin de evitar la agudización de los procesos de exclusión social.

También se hace necesario diferenciar la exclusión social, con lo que entendemos por inclusión social y educativa. En este sentido, es relevante la explicación que hace Rubén Lo Vuolo (1995) en relación con las categorías de inclusión y exclusión:

La exclusión se entiende como todas aquellas condiciones que permiten, facilitan o promuevan que ciertos miembros de la sociedad sean apartados, rechazados o simplemente se les niegue la posibilidad de acceder a los beneficios institucionales. De modo contrario, [...] la inclusión social significa englobar al conjunto de la población en el sistema de instituciones sociales, concierne tanto al acceso a sus beneficios, como a la dependencia del modo de vida individual con respecto a los mismos. (p. 15)

Ambas dinámicas se amplían en tiempos de desigualdad y segmentación social. Esto ha dado lugar a la profundización de los procesos de diferenciación social con su consecuente correlato en la declinación del acceso y permanencia a la educación, que si bien están formalmente establecidos, no logran extenderse en condiciones de vulnerabilidad social. Tal situación ha desafiado al Estado, quien plantea nuevas formas de inclusión educativa destinadas a grupos focales, en los que son visibles la pobreza y la marginalidad (Gentili, 2001). 


\section{Planes educativos destinados a jóvenes y adultos de nuestro territorio nacional: ¿nuevas formas de inclusión?}

La preocupación por la inclusión educativa, que ha adquirido relevancia en los últimos años, sin duda es consecuencia de un profundo proceso de fragmentación educativa vivido en nuestro país en las últimas décadas. En este tiempo hemos visto proliferar planes y programas educativos ofrecidos como alternativas educacionales por fuera del sistema escolar formal.

Si bien los procesos de exclusión educativa comienzan a partir de las décadas de los setenta y los ochenta, en el auge de los gobiernos de facto y de expansión del neoliberalismo, en la década de los noventa comienza a profundizarse la fragmentación del Sistema Educativo Nacional. Esto provoca, a nuestro juicio, un lento e ininterrumpido proceso de segmentación y diversificación de ofertas, que unido a la baja capacidad retentiva del sistema, en las poblaciones más vulnerables, ahondaron la inequidad y exclusión educativa, pese a los fallidos intentos de asistencia basados en acciones focalizadas para favorecer el reingreso de las poblaciones excluidas al sistema escolar.

En este contexto es posible identificar factores internos y externos al sistema educativo que estuvieron vinculados con los procesos de exclu- sión educativa. Entre los primeros, y sin ánimo de ser exhaustivos, se identifican la pérdida de financiamiento, la baja calidad en las prestaciones y la diferenciación territorial de las ofertas. Esto último vinculado a la transferencia de servicios a provincias con diversas capacidades de gestión, en el marco de una reforma de gestión federal, que profundizó las diferenciaciones interjurisdiccionales, sin arbitrar mecanismos compensatorios para garantizar la homogeneidad de la calidad nacional.

Entre los aspectos externos, debiéramos considerar, entre otros, la crisis económica y el desempleo que pauperizó las condiciones de muchas familias en todas las provincias, con mayor impacto en las zonas del NOA (noroeste argentino) y NEA (noreste argentino).

En este escenario, comienzan a instrumentarse una serie de políticas focalizadas, nacionales y provinciales destinadas a compensar, muchas veces mediante acciones remediales, los efectos de la pérdida de capacidad económica de los grupos más vulnerables. Entre ellos se destacan los programas y planes educativos que surgen a partir de mediados de los noventa de forma paralela a las instituciones escolares, destinados a sectores de la población que se iban desgranando del sistema escolar. Su implementación, en general, se realizó sin indagar las motivaciones que ocasionaron la huida masiva de jóvenes y adultos de las escuelas y, por ende, las modalidades educacionales tendientes a apaciguar el fenómeno 
que estaba en crecimiento no siempre fueron ajustadas. De esta manera, el impacto en la mejora tanto en la incorporación como la retención de los estudiantes no se logró, entre otros motivos por centrarse en aspectos organizativos y de gestión internos al sistema educativo, sin desarrollar una política de mejora en las condiciones del contexto familiar, social, económico que condicionan la educabilidad de las poblaciones escolares.

Políticas focalizadas, que tal como señala Pablo Gentili (2001), preocupadas por la "gente", se dirigen a incidir en los efectos de la exclusión, no en los procesos y dinámicas que la producen. De esta manera han tenido un efecto paliativo garantizando mejoras provisorias, sin modificar las condiciones estructurales que operan en la base de este proceso. La permanencia de estas acciones, por el contrario, si bien ofrecen una estrategia compensatoria, estabilizan la diferenciación creciente sin impedir la producción de nuevos excluidos, que siguen generándose debido a un modelo económico de acumulación y no distribuidor de riqueza.

De esta manera, en Argentina, como en el resto de la región, se expandió una serie de políticas y programas tendientes a incidir de manera focalizada en los resultados no deseados de la política educativa: analfabetismo, abandono y desgranamiento.

\section{En el ámbito provincial: políticas focalizadas y nuevos formatos escolares}

Como hemos explicitado desde un principio, analizaremos en esta oportunidad las políticas educativas que se han desarrollado en las últimas décadas en la provincia de San Luis, ya que en el territorio se ha adoptado la inclusión como una de las prioridades esenciales, entendiendo que la diferenciación de dispositivos escolares sería el camino para ampliar la capacidad inclusiva de poblaciones excluidas del sistema escolar y de producir propuestas de mayor calidad.

Con el fin de organizar el análisis, podríamos diferenciar los planes y programas orientados a las poblaciones que no han culminado la escolaridad (los niveles primario o secundario) y los nuevos formatos de escuela para la población estudiantil de la provincia. Respecto de esta última, si bien tienen en un principio el objeto de recuperar poblaciones que están en riesgo de deserción o que "fracasan" en las escuelas comunes, aparecen presentados en el discurso oficial, más bien como estrategias escolares de 
calidad superior a la escuela común o tradicional, que tal como expresan en su discurso, por su estructura misma se ha vuelto obsoleta.

Para nosotros, esta política ha profundizado la fragmentación y segmentación del sistema educativo provincial, que en un contexto de desigualdad social en el acceso a bienes y derechos, profundiza aún más los procesos de desigualdad y exclusión y le resta carácter integrador ${ }^{2}$.

\section{Planes y programas de recuperación de los "caídos del sistema"}

Rastreando los antecedentes de estas estrategias de la política educativa provincial tendientes a focalizar la intervención en la población que venía quedando fuera del sistema escolar, encontramos en el año 2001 el plan de alfabetización denominado Bandera Blanca. El plan, impulsado por el Estado provincial, estaba destinado a alfabetizar a adultos que no habían iniciado o finalizado su educación básica. El objetivo principal era enseñar a leer, escribir y las operaciones de cálculo básicas.

El plan de alfabetización se desarrolló a partir del año 2001 hasta mediados del año siguiente. Terminado el mismo, durante más de seis años, no se conformaron en la provincia otras ofertas educativas

2 Cabe aclarar que en la provincia de San Luis el sistema educativo formador de jóvenes y adultos del nivel primario se cerró definitivamente en el año 1995 argumentando escasez de matrícula (Decreto N.o 403). Argumento que no coincide con los datos estadísticos. destinadas a la población adulta. Es menester aclarar que dicha instancia alfabetizadora no planteaba posibilidades reales para que los adultos pudieran continuar sus estudios básicos en las instituciones escolares de la provincia. En primer término porque era, y aún es, inexistente una oferta educativa (dentro del sistema educativo formal), tanto de gestión pública como privada, que les posibilite a los adultos la culminación de sus estudios primarios (actualmente seis años de formación para el nivel). En segundo término porque la certificación que se les otorgaba solo acreditaba la aprobación del curso de alfabetización, es decir, no implicaba la aprobación de un año o de un ciclo de lo que actualmente es el nivel primario (Ley Nacional de Educación N. ${ }^{\circ}$ 26.206).

Recién en el año 2008 en la provincia se sancionó la ley ${ }^{3}$ que establecía "crear el Plan de Experiencia Educativas e Itinerarios Formativos" para niños, jóvenes y adultos, mediante la aplicación de proyectos innovadores, con la finalidad de garantizar la educación obligatoria (evidencia que no se estaba garantizando entonces), una mayor calidad en la educación, la remoción de barreras para el ingreso o la permanencia en el mundo del trabajo y la participación ciudadana.

A partir de junio del año 2008, en el marco de dicha ley, funcionó en la provincia un proyecto educativo "innovador" denominado Entre Clases.

3 Ley N. II -0606-2008. 
El proyecto básicamente convocaba a jóvenes y adultos de la provincia de San Luis, sin estudios primarios y secundarios completos, a concurrir a los centros educativos seleccionados para tal fin.

En ambos planes se evidencia la necesidad de reescolarizar a las poblaciones adultas que no han logrado graduarse en la edad prevista, lo que evidencia que no se garantiza la universalidad educativa y refleja la necesidad de compensar por fuera del Sistema Educativo Nacional y Provincial, de ahí que se puede decir que en los últimos quince años "la entrada y la permanencia efectivas en el nivel primario distaron de ser universales" (Brito \& Stagno, 2010, p. 6). Indudablemente el sistema educativo ya no estaba alojando a la mayoría de la población, al menos en sistemas equivalentes en cuanto a la prestación curricular y a los saberes distribuidos.

Si bien estos programas ofrecieron una salida para favorecer la terminación de los estudios a los adultos, la oferta curricular y pedagógica que se aplicó no fue equivalente en calidad a la oferta del nivel, por lo cual, la prestación curricular y los saberes distribuidos reprodujeron la diferenciación en las competencias que garantizaban para estas poblaciones.

En el año 2012, el Estado Provincial lanzó un nuevo plan de terminalidad educativa destinado a jóvenes sanluiseños de entre 20 y 30 años (Ilamado Plan 20/30) con sus estudios primarios completos y que no hayan concluido sus estudios secundarios. Tenía como objetivo ofrecer la terminalidad del nivel secundario a jóvenes de la Provincia de San Luis, en el rango de edad señalado, quienes podrían terminar sus estudios secundarios, contando con el pago de una beca mensual para poder realizarlo. El cursado del plan se realizó en las Escuelas Públicas Digitales con una modalidad basada en una plataforma virtual de enseñanza y en el acompañamiento de un moderador que colaboraba en el manejo de las nuevas tecnologías y un tutor virtual disciplinar.

Otro de los planes que se puso en vigencia en el año 2015 para favorecer la inclusión fue el Plan de Inclusión Educativa (PIE) aprobado por la Ley N. ${ }^{\circ}$ II-0911-2014. En dicho documento se explicita:

Crear el Plan de Inclusión Educativa en el ámbito del Sistema Educativo Provincial, cuyo objetivo será la terminalidad de los niveles de educación Primaria y Secundaria [...]. Aplicar al Plan de Inclusión Educativa la modalidad de Escuela Pública Digital [...] Serán objetivos del Plan de Inclusión Educativa: a) Completar los niveles de educación primaria y secundaria; b) Brindar un espacio 
de aprendizaje innovador accesible a todos los nativos o habitantes de la provincia de San Luis; c) Contener socialmente a todo ciudadano que se encuentra fuera del sistema educativo formal (Artículos 1-4).

El mismo logra una cobertura muy amplia, contando en pocos meses con más de 45000 sanluiseños destinatarios, reúne características muy similares a las otorgadas al plan anterior: está destinado a jóvenes que no han finalizado sus estudios primarios o secundarios (a partir de los quince años), la modalidad es la que se desarrolla en las escuelas digitales de la provincia de San Luis. Es decir, se trata de una modalidad de cursado semipresencial, con seguimiento virtual de los aprendizajes por parte de tutores, tanto en el uso de las nuevas tecnologías como en el manejo teórico de los conocimientos que circulan en los encuentros, el pago de una beca mensual, entre otros.

\section{Dos formas específicas de segmentación educativa: Escuelas Autogestionadas y Escuelas Públicas Digitales}

Hasta aquí hemos descrito una serie de planes y programas de la política educativa de la provincia de San Luis, que han estado orientados a incidir de manera focalizada en determina- das poblaciones que básicamente se han "caído del sistema escolar". Otro fenómeno que a nuestro criterio ha contribuido a segmentar el sistema escolar es la creación de "nuevos formatos escolares", que conviven con las escuelas "comunes". Nos referimos específicamente a las Escuelas Públicas Autogestionadas y a las más recientes Escuelas Públicas Digitales.

Si bien ambos formatos escolares surgen en periodos históricos diferentes, con coyunturas histórico-políticas diferentes, a saber, las Escuelas Autogestionadas a fines de la década de los noventa y las Escuelas Públicas Digitales en el año 2011. Su creación se enmarca en el discurso oficial, en la necesidad de crear instituciones que sean capaces de adecuarse a los nuevos tiempos.

Las escuelas públicas Autogestionadas modifican la modalidad de administración y gestión de la institución escolar. La modalidad de estas instituciones es adoptada de las Ilamadas escuelas charter, modelo de escuela estadounidense, que se caracteriza principalmente por la transferencia, por parte del Estado, de establecimientos para que sean administrados por particulares, con lo cual las plantas funcionales se tercerizan y se otorga un subsidio. Ello representó un claro avance hacia un modelo de mercantilización del sistema público de enseñanza, una experiencia única en Argentina.

Desde el año 1999 se observan distintas iniciativas del Ejecutivo provincal para su creación. El primer intento fue a través de un proyecto 
de ley llamado Escuelas 2001, presentado al Legislativo en abril de 1999, pero que por la resistencia que generó entre docentes y padres no fue finalmente aprobado. Por lo tanto, su creación se estableció por medio de dos decretos del Ejecutivo: el 2562 (1999) y el 2342 (2002). Ya en el año 2004 el Legislativo terminó sancionando la ley que les dio marco y los regula (5692).

Esta ley habilita al poder ejecutivo para crear escuelas nuevas bajo esta modalidad y para la reconversión de escuelas ya existentes, cuya incorporación se debe realizar a través de convocatoria pública. Así lo explicita la Ley N 5692:

"El Poder Ejecutivo podrá declarar experimental una escuela con la finalidad de lograr una mayor calidad en la educación, fijando en el mismo acto administrativo planes, programas y metodologías" (artículo 1).

La concesión se otorgó a asociaciones educacionales, asociaciones civiles sin fines de lucro, responsables de organizar el proyecto pedagógico, de gestionar la institución, de los resultados obtenidos y de la administración financiera.

El financiamiento de estas instituciones queda garantizado por el Estado y para ello se determina una asignación por alumno USE (unidad de subvención escolar) que es entregada mensualmente a la institución. La USE básica correspondiente a la provisión de las horas mínimas requeridas varía de acuerdo al nivel educativo, a la zona que se establezca para cada establecimiento y a la provisión o no del edificio por parte del Estado (Pelayes, 2011).

Este proceso, que ha dado lugar a la existencia en la provincia de doce escuelas experimentales autogestionadas, ha producido una profunda segmentación en la propuesta curricular, en el número de horas de clase, perfiles docentes, formas de organización del tiempo y el espacio escolar, en relación con las denominadas "escuelas comunes".

El otro modelo, que nos interesa presentar es el de las "Escuelas Públicas Digitales". Las mismas nacen en el año 2010, a partir de la sanción de la Ley II- 0738-2010, que establece las bases de su funcionamiento y habilita a asociaciones no gubernamentales, asociaciones civiles y privadas y fundaciones a crear escuelas de este tipo, bajo los requerimientos que la misma ley establece.

Citamos textualmente del proyecto la definición de las Escuelas Públicas Digitales:

Se considera Escuela Pública Digital al sistema educativo que incorpore a las tecnologías de la información y la comunicación como vehículo para la construcción del 
aprendizaje. Que tendrá como objetivo beneficiar a la población en la incorporación y generación de contenidos; como así también aptitudes que aporten a la creatividad, productividad y libertad de pensamiento para lograr una continua evolución en el contexto actual de la sociedad del conocimiento. (Artículo 1.०)

Esta nueva tipología de escuela surge en un contexto de cargada conflictividad, entre los sectores docentes, gremiales y el gobierno de la provincia, fundados básicamente en viejos reclamos (sin resolverlos adecuadamente) sobre las condiciones del trabajo docente (salarios dignos, estabilidad, cobertura de cargos) ${ }^{4}$.

Entre los argumentos para su creación, se ha puesto de relieve la caducidad del sistema educativo "tradicional". Por su parte los formatos de las escuelas analizadas vendrían a optimizar la calidad de la educación pública de la provincia, otorgando posibilidades de inclusión social, tecnológica y educativa que garanticen igualdad de oportunidades. Se argumenta desde el discurso oficial que las escuelas vendrían a proporcionar una opción educativa

4 Es importante mencionar que este año (2010) un $90 \%$ de los docentes de la provincia sostuvieron un paro que se extendió por un mes. Varios medios nacionales y provinciales informaron y tomaron posición sobre este hecho. Para más información ver: Diario La Nación, 23/0371011/04/10; Diario Clarín: 29/03/10. Diario Crónica de San Luis (diario digital) 15/03/10. de calidad que contemple al alumno como individuo único y con necesidades específicas, cuyo potencial solo puede encontrar el límite en la excelencia definida como el mayor desempeño personal en todos los aspectos.

No es un dato menor que las escuelas sean definidas en los documentos oficiales como el sistema educativo que incorpora las tecnologías de gestión, de información y la comunicación, como vehículo para la construcción de los aprendizajes, que tendrá como objetivo la incorporación y generación de contenidos, así como el desarrollo de aptitudes necesarias para lograr una continua evolución hacia la sociedad del conocimiento. Claramente observamos en el párrafo anterior que las escuelas están pensadas como instituciones que organizan un nuevo sistema educativo provincial. Como bien lo establece el proyecto de EPD, las nuevas tecnologías serían los objetos materiales por excelencia que darían identidad a las prácticas de enseñanza y aprendizaje. A continuación citamos el artículo que hace referencia a lo que venimos sosteniendo:

"El sistema se implementará a través de la creación de Escuelas Digitales (ED) cuya gestión podrá ser provincial, municipal o social; la cual se hará cargo de proporcionar la locación, como así también su mobiliario, mantenimiento y limpieza" (artículo 5).

Esta experiencia, basada en el requerimiento de favorecer la alfabetización digital se ha complementado 
con programas nacionales tipo Conectar Igualdad, y otros, basados en el programa 1 a 1 de Negroponte, que ha tenido mucha difusión y extensión en la región y que lejos de perder vigencia logra cada día más visibilidad en las políticas educativas.

Sabemos que la alfabetización digital debería ayudar a promover lecturas (y escrituras) sobre la cultura que portan las nuevas tecnologías, que les permitan a los sujetos "entender los contextos, las lógicas y las instituciones de producción de saber de esos saberes [...] y que los habiliten a pensar otros recorridos y otras formas de producción y circulación" (Dussel, 2007, p. 14).

\section{Reflexiones finales}

Las estrategias que han sido puestas en práctica como formas de abordar las dificultades para el ingreso y permanencia dentro del sistema educativo de las poblaciones más vulnerables (planes y programas educativos y nuevos formatos escolares) aparecen justificadas en los discursos de los Estados, organismos internacionales y académicos desde la necesidad de incluir, vista como una de las estrategias necesarias para superar los grandes problemas de exclusión y desigualdad que producen y reproducen las sociedades contemporáneas y los mismos sistemas educativos.

En este sentido, la estrategia principal para incluir a las poblaciones escolares en Argentina y los países de la región han sido las políticas tendientes a fortalecer, mediante acciones remediales, la universalización de la escolaridad básica, lo cual estaría indicando, tal como argumentan los gobiernos de corte neoliberal, una disminución de los índices de exclusión educativa. La otra estrategia, tal como se mencionó, ha sido la de focalizar las políticas en los "efectos de la exclusión", es decir en el analfabeto, en el que abandonó la escuela. Esto tiene efectos de poco alcance, principalmente porque no incide en los procesos que producen la exclusión (Gentili, 2001).

Estas estrategias, que en la Argentina y específicamente en la provincia de San Luis, se materializan en los planes y programas "para finalizar los estudios" en periodos acotados, y en la creación de nuevos formatos escolares, representan alternativas inclusivas para los sectores vulnerables, y por ende se reconocen sus ventajas en la escolarización. Sin embargo, no han podido solucionar los aspectos de la oferta curricular que representa menor calidad educativa. Por lo tanto, al hablar de segmentación nos referimos precisamente a la existencia de circuitos diferenciados según cali- 
dad de la oferta y en función del nivel socioeconómico y socio-educativo de los sectores escolares.

Con lo cual la escolarización y la educabilidad son factores indispensables al momento de identificar la capacidad de las ofertas, no solo para incluir sino para integrar a las personas a condiciones de calidad que garanticen una vida social equivalente.

Segmentación de la oferta educativa de los diferentes niveles, que refuerzan aún más las desigualdades, tanto en la cantidad de conocimientos que las escuelas abordan como en su calidad. Creemos que el proceso de segmentación educativa provocó en un breve periodo la fragmentación del sistema educativo.

Claramente sabemos que la escuela como institución social se ve interpelada por nuevas exigencias, mandatos, desafíos; quizá en el fondo este escenario refleje un claro debilitamiento de sus funciones.

Esta segmentación, estimamos, es justamente la forma de aquel tercer tipo de exclusión que mencionaba Castel, es decir la exclusión que incluye a los grupos en circuitos de relaciones sociales y acceso a bienes de intercambio materiales y simbólicos desjerarquizados y que justamente por mantener la ilusión de la "inclusión" de los mismos, invisibiliza el mantenimiento de las condiciones estructurales que producen la exclusión y la desigualdad.

Hablar de inclusión educativa, entonces, como contracara de estas formas de inclusión pero con baja capacidad integrativa en cualquier escenario del que se trate (escuelas, universidades, institutos de formación superior), remite a pensar en estrategias políticas que complementen estas medidas mediante acciones que modifiquen los procesos económicos que producen la pobreza y sus consecuencias.

De allí que sea necesario implementar acciones que incluyan a los diversos en políticas educativas que garanticen inclusión y capacidad integrativa en las múltiples opciones educativas que hacen posible la heteronormatividad. Pero, ¿qué es lo diverso? ¿Qué sentidos asume en el escenario antes descrito? Si bien, en principio, asumir lo diverso parece ser un paso en el reconocimiento del otro, con su propia singularidad, tal vez se corra el riesgo de distanciarse de lo común, que es también lo que permite la construcción de un universo compartido del cual se pueda formar parte.

Sostenemos que estas ofertas educativas diversas han actuado como 'tranquilizadoras' de situaciones de desigualdad y exclusión en crecimiento; estos planes han ofrecido paliativos de ingreso y retención para las poblaciones más vulnerables, pero no han logrado integrar, sino que han actuado como sostén de una situación de diferenciación, producto de la pobreza y precariedad educativa que se refuerza en los noventa y que continúa en la actualidad.

Además, al margen de la importancia que en la política de integración planteada puedan representar 
estas reformas orientadas a diversificar modelos organizacionales de escuelas para poblaciones objetivo, el centro del debate debiera ser garantizar el cumplimiento de su misión, para lo cual es indispensable que la escuela funcione como unidad educativa, inclusiva, para todas las diversidades poblacionales en condiciones de ser atendidas, con resultados al concluir el nivel educativo (preescolar, primaria o secundaria) sobre todo en el campo del desarrollo de las habilidades intelectuales y de las actitudes producto de la combinación del trabajo en experiencias formativas de calidad.

Que la escuela funcione como unidad significa, al decir de Furlan y Hargreaves (2000), que logre programas conjuntos de calidad, trabajo en equipo y quiebre de estructuras balcanizadas que dificultan la integración y generan individualismo y separación en los programas que obstruyen las formas colaborativas y la inclusión de la diversidad de grupos que operan en la sociedad.

Además de estos aspectos, propios de los sistemas de organización y gestión, consideramos que la mejora de la inclusión en el sistema, debiera debatir sobre cuáles son los conocimientos valiosos que deberían circular en la escuela; qué sujetos debemos formar en las instituciones escolares; qué vinculaciones debería generar la escuela con otras instituciones sociales para aunar esfuerzos en la formación y cómo insertar la política educativa en un plan integrador de desarrollo social y humano coordinado entre las prestaciones y jurisdicciones existentes.

\section{Referencias bibliográficas}

Birgin, A. (2004). La escuela en el contexto de las trasformaciones del trabajo y de los procesos de inclusión social. En: Claudia Jacinto (coord.) ¿Educar para qué trabajo? Discutiendo rumbos en América Latina (pp. 353-364). Buenos Aires, redEtis (IIPE-IDES) MTE Y SS MECYT/La Crujía.

Brito \& Stagno (2010). La desigualdad social y cultural en la escuela: reflexiones en torno a una compleja tensión. Clase 17 perteneciente a la Diplomatura Superior en Currículum y Prácticas Escolares en Contexto. Buenos. Aires. Flacso virtual, Argentina.

Castel, R. (1997). Las metamorfosis de la cuestión social. Una crónica del salariado. Argentina: Paidós.

Corti, A. M. (2000). La vinculación entre educación y trabajo a finales del milenio. Innovación Educativa, 10, 133-139. 
Corti, A. M. (2008). La política educativa y la fragmentación social en la Argentina. En: Ana M. Corti (comp. y autora). Regulación social y políticas educacionales en América Latina. E-Book, Ediciones LAE, Laboratorio de Alternativas Educativas, UNSL. Argentina. Marzo 2008. ISBN 978-1504 8.

Dussel, I. (2007). El currículum: aproximaciones para definir qué debe enseñar la escuela hoy. Fascículo 7. Ministerio de Educación de la Nación Argentina: Buenos Aires. Echeita Sarrionandia, G. \& Duk Homad, C. (2008). Inclusión educativa. En Reice. 6 (2), 1-8.

Furlan \& Hargreaves. (2000). La Escuela que queremos. Los objetivos por los que vale la pena luchar. México: Ed. Amorrortu.

Gentili, P. (2001). La exclusión y la escuela. El apartheid educativo como política de ocultamiento. Docencia, 15, 4-11.

Gentili, P. (2011). Adentro y afuera. El derecho a la educación y las dinámicas de exclusión escolar en América Latina. En: P. Gentili, Políticas, movimientos sociales y derecho a la educación (9-32), Buenos Aires: Consejo Latinoamericano de Ciencias Sociales Clacso.

Giddens, A. (1995). La constitución de la sociedad. Bases para la teoría de la estructuración. Buenos Aires: Amorrortu.

Kessler, G. (2004). Sociología del delito amateur. Argentina: Ed. Paidós.
Lo Vuolo, R. (1995).Contra la exclusión. La propuesta del ingreso ciudadano. Buenos Aires: Ed. Miño y Dávila.

Pelayes, O. (2011). Las políticas neoliberales en la provincia de San Luis. Reinvención del Estado y de la educación. Revista Argonautas 1, 165-177.

Piola, M. E. (2000, $1 .^{\circ}$ de agosto). Paradigmas en crisis ante los Nuevos y Viejos desafíos de la cuestión social en América Latina. Revista Electrónica de Geografía y Ciencias Sociales: Scripta Nova 69(80). Disponible en: www.ub.es/geocrit/sn-69-80. $\mathrm{htm}$

Sierra, F. (1998). Función y sentido de la entrevista cualitativa en investigación social. En J. Galindo Cáceres (coord.). Técnicas de investigación en sociedad, cultura y comunicación (pp. 277345). México: Pearson, Addison Wesley Longman.

Strauss, A. L. (1987). Qualitative analysis for social scientists. New York: Cambridge University Press.

\section{Documentos}

Decreto N. ${ }^{\circ} 403$ MP, 1998.

Decreto N. ${ }^{\circ} 2562$ MP, 1999.

Decreto N. ${ }^{\circ} 2342$ MP, 2002.

Decreto N. ${ }^{\circ}$ 5826-MP, 2004.

Ley Nacional de Educación N. ${ }^{\circ}$ 26.206, año 2006.

Ley Provincial N. ${ }^{\circ}$ 5692, año 2004.

Ley II: Plan de Experiencias Educativas e Itinerarios Formativos "Entre Clases". 
0738, año 2010.

Proyecto de Terminalidad de Educación Básica para Adultos a Distancia.

Programa Nacional de Alfabetización y Educación Básica para Jóvenes y Adultos.

Plan Nacional de Finalización de Estudios Primarios y Secundarios (FinEs)

Plan de Terminalidad Educativa 20/30, 2012.

Plan de Inclusión Educativa (PIE, 2015). 